\title{
TAMSULOSIN-INDUCED PHOTOSENSITIVITY RASH
}

Singapore Med J 2018; 59(6): 336-337 https://doi.org/10.11622/smedj.2018072

Dear Sir,

Tamsulosin is an alpha-1 adrenergic receptor blocker used to treat benign prostatic hyperplasia. Dizziness and headache are among the most common of its side effects. ${ }^{(1)}$ Although cutaneous drug eruption has been reported with other alpha-1 adrenergic receptor blockers, ${ }^{(2-5)}$ drug rash due to tamsulosin is relatively uncommon. (1) We herein report a case of photosensitivity rash secondary to tamsulosin.

Our patient was a 75-year-old Malay man who presented to our institution in August 2015 with a rash over his face, neck, upper chest, and upper and lower limbs. The rash had first appeared in his lower limbs two weeks earlier. It then spread to involve his upper limbs, upper chest, and finally his neck and face. The rash was itchy but without vesicle or pain. There was no preceding fever or any infective symptoms. He felt that sunlight had not worsened the rash in the sun-exposed areas of his skin.

A week before, the patient had consulted a general practitioner and had been prescribed a course of amoxicillin-clavulanic acid, the antihistamines chlorpheniramine and cetirizine, as well as a moisturising cream (Atoderm). Despite the treatment, the rash did not improve. His other new medications included analgesic paracetamol, etoricoxib and ketoprofen plaster, which were prescribed recently by a tertiary hospital for musculoskeletal pain. There was no other consumption of over-the-counter or traditional medicine. He had not come into contact with any new shampoo or potential irritants and had no known drug or food allergy.

At the time of presentation, the patient's medical history included ischaemic heart disease, chronic obstructive pulmonary disease, past history of treated pulmonary tuberculosis in the 1970s, peptic ulcer disease with previous laparotomy, inguinal hernia that had been surgically repaired and benign prostatic hyperplasia. His chronic medications included aspirin, simvastatin, ipratropium inhaler, budesonide/formoterol inhaler, famotidine, tamsulosin, lactulose and senna. He also had a supply of emulsifying ointment and betamethasone valerate $0.025 \%$ cream, which had previously been prescribed by a polyclinic doctor.

On examination, the patient was comfortable at rest. He was afebrile, normotensive and not tachycardic (blood pressure of 128/85 mmHg and pulse rate of 79 beats per minute). Examination of his skin revealed a sharply demarcated erythematous maculopapular rash distributed over his neck and upper chest in a shawl distribution, and over his distal upper and lower limbs with sparing of the interdigital areas, axilla, abdomen and the upper thighs (Fig. 1). There was no pustule, vesicle, or oral or genital ulcer. There was also no periorbital or uvula oedema. Examination of the cardiorespiratory, gastrointestinal and neurological systems was otherwise unremarkable. Upon investigation, his white cell count was raised at $12.23 \times 10^{9} / \mathrm{L}$ with predominant eosinophilia $\left(2.09 \times 10^{9} / \mathrm{L}\right)$. Review of previous full blood counts done at the polyclinic revealed persistent eosinophilia since March 2015 , at $1.15 \times 10^{9} / \mathrm{L}$ in March 2015 and $1.39 \times 10^{9} / \mathrm{L}$ in August 2015. The patient's haemoglobin and platelet counts, renal function, electrolytes and liver function tests were normal. His erythrocyte sedimentation rate was mildly elevated at $67 \mathrm{~mm} / \mathrm{hour}$, though his antinuclear antibody and anti-extractable nuclear antibody panel were normal.

Given the distribution of the rash over the sun-exposed areas, our impression was that of a possible photosensitivity rash. Further questioning revealed that the patient had been having intermittent episodes of similar rash over the preceding one year or so, which had been treated by the polyclinic doctor with steroid cream. Review of his medication history showed that among his chronic medications, oral tamsulosin was the latest to be added, in December 2014. As the commencement date of the tamsulosin closely coincided with the first onset of his rash, we felt that it was the likely culprit of the photosensitivity rash. We stopped the patient's tamsulosin and started him on clobetasol $0.05 \%$ ointment for his limbs and betamethasone valerate $0.05 \%$ ointment for his neck. His rash improved markedly over the next one week. His eosinophil count also improved correspondingly, from $2.09 \times 10^{9} / \mathrm{L}$ on admission to $0.76 \times 10^{9} / \mathrm{L}$ prior to discharge. The patient did not develop any urinary obstructive symptoms or retention despite the cessation of tamsulosin. On discharge, we were able to reduce his steroid creams to betamethasone $0.05 \%$ for the trunk and limbs, and betamethasone $0.025 \%$ for the face and neck.

The patient was reviewed in the clinic one month later. By then, his rash had resolved and hence his steroid creams were stopped. Over the next few months, he was restarted on betamethasone $0.025 \%$ cream for some residual rashes, which was then gradually tapered off. When he returned to the clinic in February 2016, he had stopped his betamethasone cream for two weeks. His rash had completely resolved with no further recurrence.

Drug-induced photosensitivity cutaneous reaction is initiated once the culprit drug comes into contact with light and is activated. However, this is only momentary, as the activated drug then loses its energy and reverts to its base state. This process can, in return, elicit a toxic or an allergic reaction, termed phototoxic reaction and photoallergy reaction, respectively. ${ }^{(6)}$

Tamsulosin-induced photosensitivity rash has only rarely been reported in the literature. ${ }^{(7,8)}$ We hope that our report will raise more awareness among physicians regarding this uncommon cutaneous reaction of tamsulosin.

Yours sincerely,

Chin Kwok $\underline{T a n}^{1}$, Keng Bee $\underline{\text { Yap }}^{1}$

${ }^{1}$ Geriatric Medicine, Department of Medicine, Ng Teng Fong General Hospital, Singapore. chin_kwok_tan@nuhs.edu.sg 

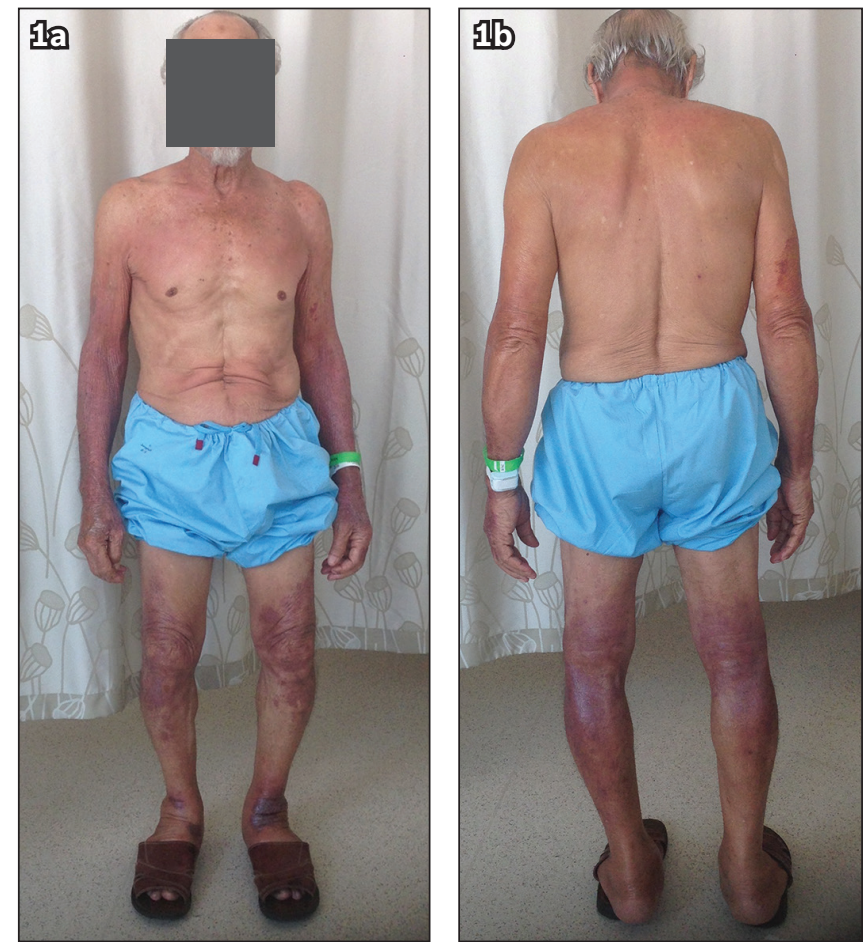

Fig. 1 Photographs of the (a) front and (b) back view of the patient show a sharply demarcated erythematous maculopapular rash distributed over his neck and upper chest in a shawl distribution, and over his distal upper and lower limbs with sparing of the interdigital areas, axilla, abdomen and the upper thighs.

\section{REFERENCES}

1. Mann RD, Biswas P, Freemantle S, Pearce G, Wilton L. The pharmacovigilance of tamsulosin: event data on 12484 patients. BJU Int 2000; 85:446-50.

2. Higashi N, Kume A, Ueda K, Ikushima M, Hino H. Photosensitive drug eruption due to cardenalin (doxazosin mesilate). Skin Res 1997; 39 : 256-9.

3. Wang YS, Tay YK, Kwok C. Toxic epidermal necrolysis caused by alfuzosin, an alpha1-adrenoceptor antagonist. Arch Dermatol 2006; $142: 938$.

4. Takehara Y, Igawa K, Satoh T, Yokozeki H. Psoriasiform eruption induced by alpha1-adrenergic blocker, urapidil. J Eur Acad Dermatol Venereal 2007; 21:577-8.

5. Inoue A, Sawada Y, Ohmori S, et al. Maculopapular type drug eruption caused by silodosin. Allergol Int 2016; 65:219-20.

6. Harber LC, Baer RL. Pathogenic mechanisms of drug-induced photosensitivity. J Invest Dermatol 1972; 58:327-42.

7. Aizu T, Kaneko T, Shiraishi M, Nomura K. Phatoallergic drug eruption caused by tamsulosin hydrochloride. Rinsho Derma 1999; 41:64-5.

8. Kamide R. Photosensitive dermatosis caused by extrinsic photosensitizing substance. In: Tamoki K, ed. New Dermatology Vol 16. Nakayama Shoten 2003: 293-300. 